\title{
Application of novel solid phase extraction-NMR protocols for metabolic profiling of human urine
}

\author{
Daniel McGill, (D) *a Elena Chekmeneva, ${ }^{a}$ John C. Lindon, (D) a \\ Zoltan Takats ${ }^{a}$ and Jeremy K. Nicholson ${ }^{b}$
}

Received 2nd December 2018, Accepted 6th December 2018

DOI: $10.1039 / \mathrm{c} 8 \mathrm{fd} 00220 \mathrm{~g}$

\begin{abstract}
Metabolite identification and annotation procedures are necessary for the discovery of biomarkers indicative of phenotypes or disease states, but these processes can be bottlenecked by the sheer complexity of biofluids containing thousands of different compounds. Here we describe low-cost novel SPE-NMR protocols utilising different cartridges and conditions, on both natural and artificial urine mixtures, which produce unique retention profiles useful for metabolic profiling. We find that different SPE methods applied to biofluids such as urine can be used to selectively retain metabolites based on compound taxonomy or other key functional groups, reducing peak overlap through concentration and fractionation of unknowns and hence promising greater control over the metabolite annotation/identification process.
\end{abstract}

\section{Introduction}

Structure elucidation is a necessary and complex task for synthetic chemistry, drug discovery, and natural products research, but it is also a major challenge in areas related to the life sciences. Metabolite structure elucidation is considered a bottleneck in metabolic profiling (the study of low molecular weight metabolite patterns in organisms) - metabolites found within biofluids can be identified and described as biomarkers characteristic of specific phenotypes and disease states. For example, Elliott et al. ${ }^{1}$ were able to characterise the biomarkers of adiposity in US adults through analysis of urine samples. Metabolites representative of different metabolite classes and biochemical pathways (such as $N$-acetylneuraminate and trimethylamine) were shown to have significant association with BMI. Hence, structure elucidation for metabolic profiling has been demonstrated to indirectly deepen knowledge of metabolic pathways, which may aid the

\footnotetext{
${ }^{a}$ Division of Computational and Systems Medicine, Department of Surgery and Cancer, Faculty of Medicine, Imperial College London, South Kensington Campus, London SW72AZ, UK. E-mail: d.mcgill16@imperial. ac.uk

${ }^{b}$ The Australian National Phenome Center, Research and Innovation, Murdoch University, 50, South Street Murdoch, Perth WA6150, Australia
} 
development of future diagnostic and therapeutic techniques. Within metabolic profiling, ${ }^{1} \mathrm{H}$ Nuclear Magnetic Resonance (NMR) spectroscopy has become an extremely valuable tool for the characterisation of complex mixtures; ${ }^{2}$ statistical spectroscopy tools have also been applied to extract information from complex spectral sets. ${ }^{3}$ Posma et $a{ }^{4}{ }^{4}$ have demonstrated the use of statistical 2D NMR, utilising the RED-STORM probabilistic statistical spectroscopy tool on data acquired from diet-controlled human urine samples, in order to further expand the understanding of dietary biomarkers.

As such, there is currently a high interest in the identification of metabolites. Urine has found widespread use in metabolic profiling due to its non-invasive ease of collection; however, the human urinary metabolome remains only partially mapped. This is in part because of the sheer number and dynamic range of compounds within a given urine sample - and, as a corollary of this, because of peak overlap that frustrates annotation efforts. In a high-throughput NMR study of human urine, it was found by Bingol et al. ${ }^{5}$ that in a sample ${ }^{13} \mathrm{C}-{ }^{1} \mathrm{H}$ HSQC spectrum, of the 1012 peaks detected, only 437 peaks (belonging to 98 individual metabolites) could be assigned. In 2013, Bouatra et al. ${ }^{6}$ utilised a variety of analytical platforms (NMR, GC-MS, DFI/LC-MS/MS, ICP-MS, and HPLC) in order to identify 445 and quantify 378 unique urine metabolites, and a literature review led to the identification of an additional 2206 compounds. The Urine Metabolome Database (http://www.urinemetabolome.ca) at the time of writing counts 4237 total metabolites, of which only 1607 are 'detected and quantified', 421 are 'detected but not quantified', and 2209 are 'expected but not detected'.

There is a need to expand the understanding of the human urinary metabolome in order to improve our capacity for characterization of population phenotypes and for discovery of biomarkers related to disease and diet. Currently, the two most powerful analytical techniques used for metabolite annotation (the putative identification of metabolites based on spectral similarity to literature or external data) and identification (confirmation of molecular identity based on 'a minimum of two independent and orthogonal datasets relative to an authentic reference standard') 7 are mass spectrometry (MS) and NMR spectroscopy, as they provide orthogonal qualitative data, as well as absolute and relative quantification, in a very precise and high-throughput manner. In the framework of LC-MS analysis, different column chemistries have been explored to ensure wide metabolome coverage of biofluids, and to take account of the physicochemical diversity of their components. Integration of NMR spectroscopy allows acquisition of complementary information for definitive structure elucidation and confirmation. Despite its reproducibility, ease of use, and quantitative data generation, the limited sensitivity of NMR remains a bottleneck to metabolite identification - low-concentration compounds become indistinguishable from noise, and peak overlap by more concentrated compounds regularly obscures signals from less concentrated compounds.

The use of SPE-NMR of urine has been demonstrated previously by Wilson and Nicholson for retention of specific drug metabolites such as paracetamol, ibuprofen, and naproxen. ${ }^{8}$ The fractionation of human urine using SPE to reduce NMR peak overlap has also been demonstrated by Yang et al. ${ }^{9}$ and Jacobs et al. ${ }^{\mathbf{1 0}}$ using C18 and HLB cartridges, respectively. All previous SPE-NMR experiments have utilised standard or widely available methods using classic reversed phase sorbents, but have not varied or altered conditions such as $\mathrm{pH}$ to generate 
different retention profiles; additionally, the use of ion exchange SPE cartridges on biofluids for the purpose of metabolite annotation has not been attempted before. The expansion of available SPE methods should promise greater control over metabolite retention, and hence greater insight into the human urinary metabolome - this can be done through studying how different SPE methods can retain not only individual metabolites, but entire compound classes. Hence this approach is highly relevant to the detection and characterisation of unknowns in many complex biological mixtures.

\section{Experimental}

\section{Sample collection}

Urine was collected from 12 healthy volunteers in $500 \mathrm{~mL}$ Corning ${ }^{\mathrm{TM}}$ tubes prerinsed with ultrapure water - each volunteer provided informed consent in writing. Each urine sample was individually analysed using NMR as a check for polyethylene glycol contamination. Samples were pooled into a pre-rinsed polypropylene carboy with a stir bar. The pooled sample was homogenised by stirring for five minutes, after which $15 \mathrm{~mL}$ aliquots were dispensed into $20 \mathrm{~mL}$ Sterilin sample tubes. Samples were labelled sequentially and stored at $-80^{\circ} \mathrm{C}$; samples to be used were subsequently transferred to a $4{ }^{\circ} \mathrm{C}$ fridge for thawing.

\section{pH-altered urine samples}

Concentrated $\mathrm{HCl}$ or $\mathrm{NaOH}$ solution was added dropwise to $250 \mathrm{~mL}$ of pooled urine until the desired $\mathrm{pH}$ was achieved. The acidified ( $\mathrm{pH} 2$ and $\mathrm{pH} 5)$ and basified (pH 11 and $\mathrm{pH}$ 9) samples were then stored at $4{ }^{\circ} \mathrm{C}$.

\section{Artificial urine preparation}

$500 \mathrm{~mL}$ ultrapure water was added to a $1 \mathrm{~L}$ flask. Constituent compounds and salts were then added (see Results section, Table 2) with constant stirring. An additional $500 \mathrm{~mL}$ of distilled water was added to make $1 \mathrm{~L}$ of artificial urine.

\section{SPE methods}

All solid phase extraction cartridges obtained had a $6 \mathrm{~mL}$ capacity and $500 \mathrm{mg}$ bed weight. C18 cartridges were acquired from Thermofisher (Hypersep ${ }^{\mathrm{TM}}$ ) and Agilent (Bond Elut ${ }^{\mathrm{TM}}$ ). $\mathrm{C}_{18}$-ec, $\mathrm{NH}_{2}$ (Weak Anion eXchange - WAX), $\mathrm{CN}$, and $\mathrm{SO}_{3}{ }^{-}$ (Weak Cation eXchange - WCX) cartridges were acquired from Biotage (Isolute $\left.{ }^{\mathrm{TM}}\right) . \mathrm{C}_{8}$, phenyl, and diol cartridges were acquired from Thermofisher (Hypersep ${ }^{\mathrm{TM}}$ ). Hydrophilic-Lipophilic Balance (HLB) cartridges were acquired from Waters (Oasis ${ }^{\mathrm{TM}}$ ). PhenylBoronic Acid (PBA), Strong Anion eXchange (SAX), and Strong Cation eXchange (SCX) cartridges were acquired from Macherey-Nagel (Chromabond ${ }^{\mathrm{TM}}$ ). Sample pretreatment, conditioning, equilibration, wash, and elution steps were tailored for the cartridge and method. Different methods utilised a variety of $\mathrm{pH}$ levels and solvent systems (Table 1). The use of different conditions altered the retention profiles exhibited by the different cartridges, resulting in elutions composed of different metabolites.

Reversed phase cartridge $\left(\mathrm{C}_{18}, \mathrm{HLB}\right.$, and phenyl), neutral $\mathrm{pH}$ sample. A Thermofisher Hypersep ${ }^{\mathrm{TM}} \mathrm{C}_{18}$ cartridge (6 mL capacity and $500 \mathrm{mg}$ bed weight) was conditioned with methanol $(6 \mathrm{~mL})$, then equilibrated with water $(6 \mathrm{~mL})$. 
Table 1 Descriptions of individual SPE methods

\begin{tabular}{|c|c|c|}
\hline Cartridge & Method name & Modification \\
\hline \multirow{4}{*}{$\mathrm{C}_{18}$} & Neutral & No modification \\
\hline & Formic acid & $\begin{array}{l}2 \% \text { formic acid added to conditioning, } \\
\text { equilibration, wash, and elution steps }\end{array}$ \\
\hline & Weakly acidified & Sample acidified to $\mathrm{pH} 5$ \\
\hline & Strongly acidified & Sample acidified to $\mathrm{pH} 2$ \\
\hline \multirow[t]{4}{*}{ HLB } & Neutral & No modification \\
\hline & Formic acid & $\begin{array}{l}2 \% \text { formic acid added to conditioning, } \\
\text { equilibration, wash, and elution steps }\end{array}$ \\
\hline & Weakly acidified & Sample acidified to $\mathrm{pH} 5$ \\
\hline & Strongly acidified & Sample acidified to $\mathrm{pH} 2$ \\
\hline \multirow[t]{4}{*}{ Phenyl } & Neutral & No modification \\
\hline & Formic acid & $\begin{array}{l}2 \% \text { formic acid added to conditioning, } \\
\text { equilibration, wash, and elution steps }\end{array}$ \\
\hline & Weakly acidified & Sample acidified to pH 5 \\
\hline & Strongly acidified & Sample acidified to $\mathrm{pH} 2$ \\
\hline \multirow[t]{4}{*}{ SCX } & Neutral & No modification \\
\hline & Formic acid & $\begin{array}{l}2 \% \text { formic acid added to conditioning and } \\
\text { equilibration steps }\end{array}$ \\
\hline & Weakly acidified & Sample acidified to $\mathrm{pH} 5$ \\
\hline & Strongly acidified & Sample acidified to $\mathrm{pH} 2$ \\
\hline \multirow[t]{4}{*}{ SAX } & Neutral & No modification \\
\hline & Formic acid & $\begin{array}{l}2 \% \text { formic acid added to conditioning and } \\
\text { equilibration steps }\end{array}$ \\
\hline & Weakly basified & Sample basified to $\mathrm{pH} 9$ \\
\hline & Strongly basified & Sample basified to $\mathrm{pH} 11$ \\
\hline \multirow[t]{2}{*}{ PBA } & Glycine buffer & Glycine-based buffer \\
\hline & Phosphate buffer & Sodium phosphate-based buffer \\
\hline
\end{tabular}

Pooled urine $(3 \mathrm{~mL})$ was loaded onto the cartridge, which was then washed with water $(6 \mathrm{~mL})$ to eliminate interferences. The retained metabolites were then eluted with methanol $(6 \mathrm{~mL})$.

Reversed phase cartridge ( $\mathrm{C}_{18}, \mathrm{HLB}$, and phenyl), $2 \%$ formic acid (all steps), acetonitrile elution. A Thermofisher Hypersep ${ }^{\mathrm{TM}} \mathrm{C}_{18}$ cartridge $(6 \mathrm{~mL}$ capacity and $500 \mathrm{mg}$ bed weight) was conditioned with $2 \%$ formic acid in acetonitrile $(6 \mathrm{~mL})$, then equilibrated with $2 \%$ formic acid in water $(6 \mathrm{~mL})$. Pooled urine $(3 \mathrm{~mL})$ was loaded onto the cartridge, which was then washed with $2 \%$ formic acid in water (6 $\mathrm{mL}$ ) to eliminate interferences. The retained metabolites were then eluted with $2 \%$ formic acid in acetonitrile $(6 \mathrm{~mL})$.

Strong cation exchange, neutral $\mathbf{p H}$ sample. A Macherey-Nagel Chromabond ${ }^{\mathrm{TM}}$ SCX cartridge (6 mL capacity and $500 \mathrm{mg}$ bed weight) was conditioned with methanol $(6 \mathrm{~mL})$, then equilibrated with water $(6 \mathrm{~mL})$. Pooled urine $(3 \mathrm{~mL})$ was loaded onto the cartridge, which was then washed with $2 \%$ formic acid solution ( 6 $\mathrm{mL})$. Methanol (6 mL) was used to elute the first set of metabolites, followed by $5 \% \mathrm{NH}_{4} \mathrm{OH}$ in methanol $(6 \mathrm{~mL})$ for the second elution.

Strong cation exchange, $2 \%$ formic acid. A Macherey-Nagel Chromabond ${ }^{\mathrm{TM}}$ SCX cartridge ( $6 \mathrm{~mL}$ capacity and $500 \mathrm{mg}$ bed weight) was conditioned with $2 \%$ formic acid in acetonitrile $(6 \mathrm{~mL})$, then equilibrated with $2 \%$ formic acid in water $(6 \mathrm{~mL})$. Pooled urine $(3 \mathrm{~mL})$ was loaded onto the cartridge, which was then washed 
with $2 \%$ formic acid solution $(6 \mathrm{~mL})$. Acetonitrile $(6 \mathrm{~mL})$ was used to elute the first set of metabolites, followed by $5 \% \mathrm{NH}_{4} \mathrm{OH}$ in methanol $(6 \mathrm{~mL})$ for the second elution.

Strong anion exchange, neutral pH sample. A Macherey-Nagel Chromabond ${ }^{\mathrm{TM}}$ SCX cartridge (6 mL capacity and $500 \mathrm{mg}$ bed weight) was conditioned with acetonitrile $(6 \mathrm{~mL})$, then equilibrated with water $(6 \mathrm{~mL})$. Pooled urine $(3 \mathrm{~mL})$ was loaded onto the cartridge, which was then washed with $5 \% \mathrm{NH}_{4} \mathrm{OH}$ solution (6 $\mathrm{mL})$. Acetonitrile $(6 \mathrm{~mL})$ was used to elute the first set of metabolites, followed by $2 \%$ formic acid in acetonitrile $(6 \mathrm{~mL})$ for the second elution.

Phenylboronic acid, sodium phosphate buffer. A Macherey-Nagel Chromabond $^{\mathrm{TM}}$ PBA cartridge (6 mL capacity and $500 \mathrm{mg}$ bed weight) was conditioned with a solution of $1 \% \mathrm{HCl}$ in $70: 30$ water : acetonitrile $(6 \mathrm{~mL})$, then equilibrated with sodium phosphate buffer basified to $\mathrm{pH} 10$ with sodium hydroxide $(6 \mathrm{~mL})$. Pooled urine $(3 \mathrm{~mL})$ was loaded onto the cartridge, which was then washed with sodium phosphate buffer basified to $\mathrm{pH} 8.5$ with sodium hydroxide $(6 \mathrm{~mL})$. Water $(6 \mathrm{~mL})$ was used to elute the first set of metabolites, followed by a solution of $1 \% \mathrm{HCl}$ in $70: 30$ water : acetonitrile $(6 \mathrm{~mL})$ for the second elution.

\section{NMR sample preparation}

Washes and elutions were dried under nitrogen and reconstituted in ultrapure water $(3 \mathrm{~mL})$. Buffer containing trimethylsilylpropionate (TMS) as an internal reference standard was added to $540 \mu \mathrm{L}$ of reconstituted sample, as described by Dona et al. ${ }^{11} 580 \mu \mathrm{L}$ of the manually vortexed sample was then transferred into $5 \mathrm{~mm}$ SampleJet NMR racks.

Samples which required additional 2D NMR experiments were dried under nitrogen and reconstituted in $\mathrm{D}_{2} \mathrm{O}(3 \mathrm{~mL})$. TMS phosphate buffer $(60 \mu \mathrm{L})$ was added to $540 \mu \mathrm{L}$ of reconstituted sample, and $580 \mu \mathrm{L}$ of the resulting manually vortexed sample was transferred into $5 \mathrm{~mm}$ NMR tubes.

\section{NMR data acquisition}

All 1D experiments were run using a Bruker Avance III $600 \mathrm{MHz}$ spectrometer equipped with SampleJet. Samples were analysed using one-dimensional watersuppressed ${ }^{1} \mathrm{H}$ NOESY experiments at $300 \mathrm{~K}$.

Additional ${ }^{1} \mathrm{H}-^{1} \mathrm{H}$ J-resolved experiments, and 2D-NMR experiments, including ${ }^{1} \mathrm{H}-{ }^{1} \mathrm{H}$ Total Correlation Spectroscopy (TOCSY), ${ }^{1} \mathrm{H}-{ }^{1} \mathrm{H}$ Correlation Spectroscopy (COSY), and ${ }^{1} \mathrm{H}_{-}{ }^{13} \mathrm{C}$ Heteronuclear Single Quantum Coherence spectroscopy (HSQC), were utilised for metabolite annotation. The data from the 2D NMR experiments was acquired using a Bruker Avance III $600 \mathrm{MHz}$ spectrometer equipped with a cryoprobe.

\section{Data analysis}

NMR datasets were imported into MatLab using the Imperial Metabolic Profiling and Chemometrics Toolbox (IMPaCTS). ${ }^{12}$ Water $(4.26-5.50 \mathrm{ppm})$ and formate $(8.25-8.63 \mathrm{ppm})$ regions were removed from the spectra to eliminate interferences; the spectra were then normalised against the TSP region ( -0.5 to $0.5 \mathrm{ppm})$ using a probabilistic quotient normalisation function. ${ }^{13}$ Principal Component Analysis (PCA) plots were subsequently constructed with 5 principal components. 


\section{Results}

Different cartridge chemistries were utilised in order to produce unique retention profiles for different compound classes (as demonstrated in Fig. 1). All samples utilised a sample load incorporating $3 \mathrm{~mL}$ of urine as a compromise between substrate retention capacity and spectral resolution. Each elution demonstrated differing retention profiles for each method - replicates of the same method, however, had little difference between spectra. Hence the reproducibility of the SPE methods outlined here can be guaranteed.

\section{Key molecules}

Quantifying the extent of retention of different classes of compound can be done using molecules representative of a chemical class. In the Human Urine Metabolome database,${ }^{14}$ chemicals are assigned taxonomically - first to classes, then to subclasses - using the ChemOnt automated taxonomy. As the taxonomy is automated, its class structure can be utilised in order to demonstrate retention profiles for given methods.

Two separate lists of metabolites were utilised to generate a list of representative compounds. One set of metabolites was examined and ranked according to their frequency of occurrence in the human urinary metabolome, ${ }^{6}$ such that the metabolites being examined would have a significant chance of being characterised in pooled urine samples. The second set was generated from a method for producing artificial urine. ${ }^{15}$ The two lists were combined, and the resulting set of

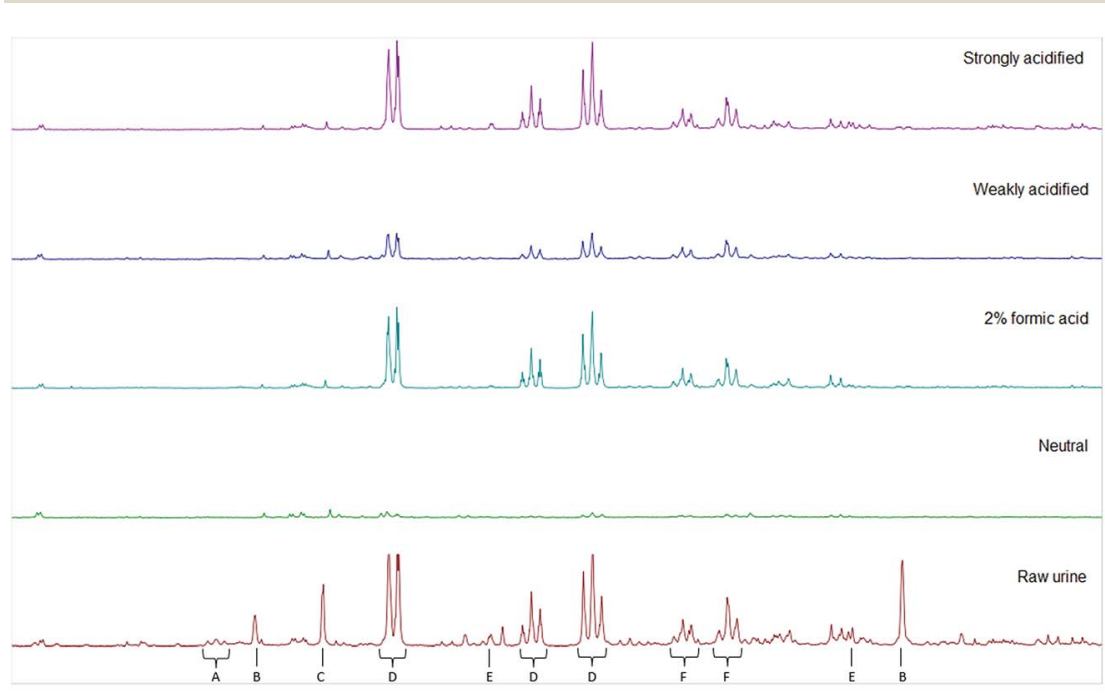

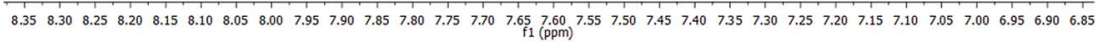

Fig. 1 A comparison of the aromatic regions of $600 \mathrm{MHz}$ NMR spectra utilising different $\mathrm{C}_{18}$ SPE methods on natural human urine. From top: sample acidified to $\mathrm{pH} 2$, sample

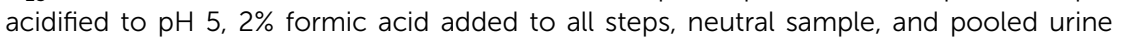
before SPE treatment. Select metabolites labelled: (A) trigonelline, (B) 3-methylhistidine, (C) histidine, (D) hippurate, (E) 2-furoylglycine, and (F) phenylalanine. 
metabolites (Table 2) was then characterised by their assigned subclass from the ChemOnt automated taxonomy.

The intensities of specific peaks corresponding to these metabolites in the elutions were compared to the intensities of the same peaks in the raw pooled urine samples. From this, the percentage retention per compound class could be

Table 2 Artificial urine mixture constituent metabolites

\begin{tabular}{|c|c|c|}
\hline Name & $\begin{array}{l}\text { Occurrence } \\
\text { in urine }(\%)^{6}\end{array}$ & Assigned subclass (HMDB) \\
\hline trans-Aconitic acid & 55 & Tricarboxylic acids and derivatives \\
\hline Tartaric acid & 82 & Carbohydrates and carbohydrate conjugates \\
\hline Succinic acid & 91 & Dicarboxylic acids and derivatives \\
\hline L-Asparagine & 95 & Asparagine and derivatives \\
\hline L-Aspartic acid & 95 & Aspartic acid and derivatives \\
\hline 2-Furoylglycine & 100 & $N$-Acyl-alpha amino acids \\
\hline 3-Aminoisobutanoic acid & 100 & Beta amino acids and derivatives \\
\hline 3-Methylhistidine & 100 & Histidine and derivatives \\
\hline Acetic acid & 100 & Carboxylic acids \\
\hline Allantoin & 100 & Imidazoles \\
\hline Betaine & 100 & Alpha amino acids and derivatives \\
\hline Citric acid & 100 & Tricarboxylic acids and derivatives \\
\hline Creatine & 100 & Alpha amino acids and derivatives \\
\hline Creatinine & 100 & Alpha amino acids and derivatives \\
\hline D-Glucose & 100 & Carbohydrates and carbohydrate conjugates \\
\hline Dimethyl sulfone & 100 & Sulfones \\
\hline Dimethylamine & 100 & Organonitrogen compounds \\
\hline Erythritol & 100 & Carbohydrates and carbohydrate conjugates \\
\hline Ethanolamine & 100 & Amines \\
\hline Formic acid & 100 & Carboxylic acids \\
\hline Glycerol & 100 & Carbohydrates and carbohydrate conjugates \\
\hline Glycine & 100 & Alpha amino acids and derivatives \\
\hline Glycolic acid & 100 & Alpha hydroxy acids and derivatives \\
\hline Guanidoacetic acid & 100 & Alpha amino acids and derivatives \\
\hline Hippuric acid & 100 & Benzoic acids and derivatives \\
\hline Indoxyl sulfate & 100 & Arylsulfates \\
\hline L-Alanine & 100 & Alanine and derivatives \\
\hline L-Cysteine & 100 & Cysteine and derivatives \\
\hline L-Cystine & 100 & L-Cysteine- $S$-conjugates \\
\hline L-Glutamic acid & 100 & Glutamic acid and derivatives \\
\hline L-Glutamine & 100 & Alpha amino acids and derivatives \\
\hline L-Histidine & 100 & Histidine and derivatives \\
\hline L-Lactic acid & 100 & Alpha hydroxy acids and derivatives \\
\hline L-Lysine & 100 & Alpha amino acids and derivatives \\
\hline L-Phenylalanine & 100 & Phenylalanine and derivatives \\
\hline L-Serine & 100 & Serine and derivatives \\
\hline L-Threonine & 100 & Alpha amino acids and derivatives \\
\hline Methanol & 100 & Alcohols and polyols \\
\hline Myoinositol & 100 & Alcohols and polyols \\
\hline$p$-Hydroxyphenylacetic acid & 100 & Phenols \\
\hline Propylene glycol & 100 & Alcohols and polyols \\
\hline Taurine & 100 & Organosulfonic acids and derivatives \\
\hline Trigonelline & 100 & Alkaloids and derivatives \\
\hline Trimethylamine N-oxide & 100 & Aminoxides \\
\hline
\end{tabular}


estimated - hence, insight into which methods can selectively retain different compound classes can be achieved. For example, the peak intensity of retained creatinine (belonging to the subclass 'alpha amino acids and derivatives') from a method utilising a $\mathrm{C}_{18}$ cartridge at neutral conditions was measured at 257 . When compared to the intensity of the same peak in the 'raw' urine sample (5027), this gives an estimated retention capacity of creatinine using this method of $5 \%$. After accounting for the other members of that subclass, $\mathrm{C}_{18}$ under neutral conditions has an overall retention of $\alpha$-amino acids and derivatives of $0.75 \%$.

The compound class retentions per method were then summed to produce a measure of the total retention capacity out of 100 - where methods ranking 0 would retain nothing, while methods ranking 100 would retain everything (Table 3). It can be expected that the sum of the retention capacity estimates of the elutions and the washes for a given method is equal to 100 .

\section{Principal component analysis (PCA)}

An alternative approach to quantifying the retention profiles of each SPE cartridge can be achieved using PCA. PCA is an analytical method that can be used to reduce the dimensionality of data and produce visual representations of correlations between datasets - for NMR spectra, it allows for clustering and trends between experiments to be demonstrated, as well as for discovering potential outliers.

A PCA structure built with the datasets from all elution methods utilising natural urine demonstrated that the elutions from acidified ion exchange methods were clearly separated from the other chemistries (Fig. 2). The below

Table 3 Total retention capacity estimates for each SPE method

SPE method

Total retention capacity (\%)

$\mathrm{C}_{18}$, neutral conditions

$\mathrm{C}_{18}, 2 \%$ formic acid

12.91

$\mathrm{C}_{18}$, weakly acidified sample

10.65

$\mathrm{C}_{18}$, strongly acidified sample

15.69

HLB, neutral conditions

8.87

HLB, $2 \%$ formic acid

17.40

HLB, weakly acidified sample

HLB, strongly acidified sample

Phenyl, neutral conditions

Phenyl, 2\% formic acid

2.05

Phenyl, weakly acidified sample

Phenyl, strongly acidified sample

SCX, neutral conditions

SCX, $2 \%$ formic acid

SCX, weakly acidified sample

SCX, strongly acidified sample

SAX, neutral conditions

SAX, $2 \%$ formic acid 

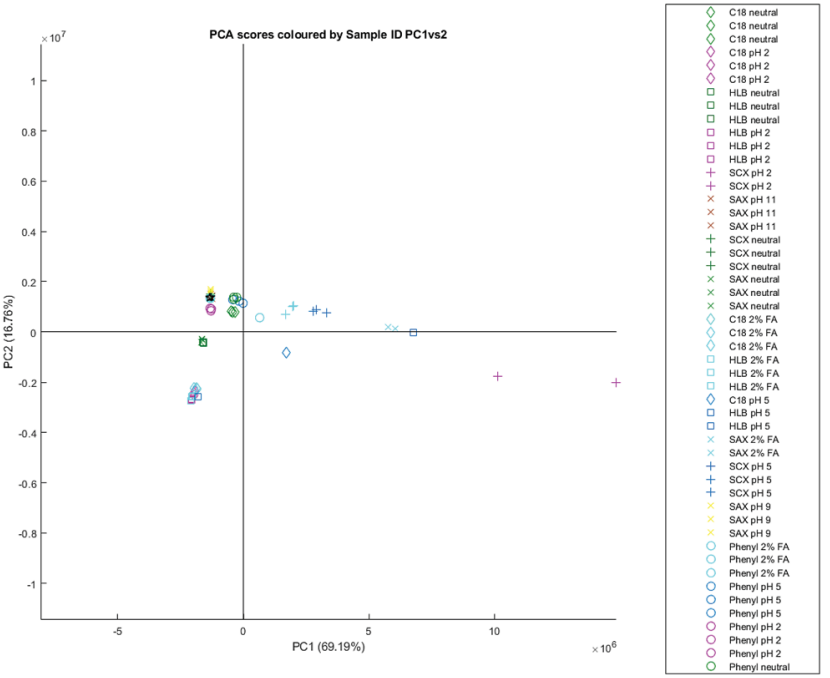

Fig. 2 A PCA scores plot based on all SPE elutions of natural urine, PC1 $(69.19 \%)$ vs. PC2 $(16.76 \%)$.

table (Table 4) lists the NMR signals visible in the PCA loadings plot, as well as their correlation coefficient and their tentative assignment - all assignments are made with comparison to the reported values in the literature, and hence can be considered annotated to a level 2 standard. ${ }^{16}$ It demonstrates that a small number

Table 4 Natural urine all elutions, PC1 assignments

\begin{tabular}{lll}
\hline Peak (ppm) & Assignment & Pearson's correlation \\
\hline $7.96(\mathrm{~s})$ & 3-Methylhistidine & 0.64 \\
$7.90(\mathrm{~s})$ & Histidine & 0.89 \\
$7.70(\mathrm{~s})$ & 1-Methylhistidine & 0.81 \\
$7.10(\mathrm{~s})$ & Histidine & 0.95 \\
$7.08(\mathrm{~s})$ & 3-Methylhistidine & 0.90 \\
$7.02(\mathrm{~s})$ & 1-Methylhistidine & 0.92 \\
$4.06(\mathrm{~s})$ & Creatinine & 1.00 \\
$4.05(\mathrm{~d})$ & Unknown A & 0.79 \\
$4.00(\mathrm{dd})$ & Histidine & 0.90 \\
$3.72(\mathrm{~s})$ & 3-Methylhistidine & 0.89 \\
$3.70(\mathrm{~s})$ & 3-Methylhistidine & 0.80 \\
$3.29(\mathrm{~s})$ & Unknown B & 0.93 \\
$3.27(\mathrm{~s})$ & TMAO & 0.96 \\
$3.25(\mathrm{~s})$ & Unknown B & 0.94 \\
$3.24(\mathrm{~s} ?)$ & Unknown C & 0.94 \\
$3.23(\mathrm{~d}))$ & Unknown D & 0.95 \\
$3.21(\mathrm{~s})$ & Unknown E & 0.84 \\
$3.18(\mathrm{~s})$ & Unknown F & 0.87 \\
$3.16(\mathrm{~s})$ & Unknown G & 0.93 \\
$3.14(\mathrm{~s})$ & Unknown H & 0.89 \\
$3.05(\mathrm{~s})$ & Creatinine & 0.99 \\
$3.04(\mathrm{~s})$ & Creatine & 0.83 \\
\hline & &
\end{tabular}


of peaks (creatinine, histidine, creatine, trimethylamine-N-oxide, and 3-methylhistidine being the most prominent) contributed to $69.24 \%$ of the differences between elutions. These metabolites were all retained by SCX cartridges under acidic conditions, and their spectral peaks are sensitive to $\mathrm{pH}$ changes, causing significant chemical shifts even in buffered samples.

Unknowns in bold have both ${ }^{1} \mathrm{H}$ and ${ }^{13} \mathrm{C}$ signals identifiable, but could not be matched to compounds in metabolite databases.

The ion exchange method most separated from other datasets is the one which produces elutions from strongly acidified urine using an SCX cartridge. However, there is also significant differentiation with the SAX elutions with $2 \%$ formic acid - under these conditions, the SAX cartridge begins to retain compounds like creatinine and histidine where it otherwise wouldn't under neutral or basic conditions. It is not clear why the SAX retention profile would begin to resemble that of SCX; one possibility could be that the silanol groups from the silica on which the $\mathrm{SO}_{3}{ }^{-}$and ammonium modifications are based are more able to retain these compounds under acidic conditions. However, silanols are usually protonated under acidic conditions, and hence would not express this ionic character; the explanation additionally doesn't account for why $\mathrm{C}_{18}$ with $2 \%$ formic acid - which, similarly, contains silanol groups - does not retain creatinine or histidine.

Besides the separation between ion exchange and reversed phase methods, there is additional separation along the secondary principal component axis between phenyl, $\mathrm{C}_{18}$ neutral, SAX neutral, and SCX neutral elutions, and $\mathrm{C}_{18}$ acidified and HLB acidified elutions - with HLB neutral elutions found in between the two clusters (Fig. 3). Removing elutions from ion exchange cartridges and reconstructing the PCA affords a similar differentiation with more clarity. The clusters suggest that $\mathrm{C}_{18}$ neutral elutions have more in common with phenyl

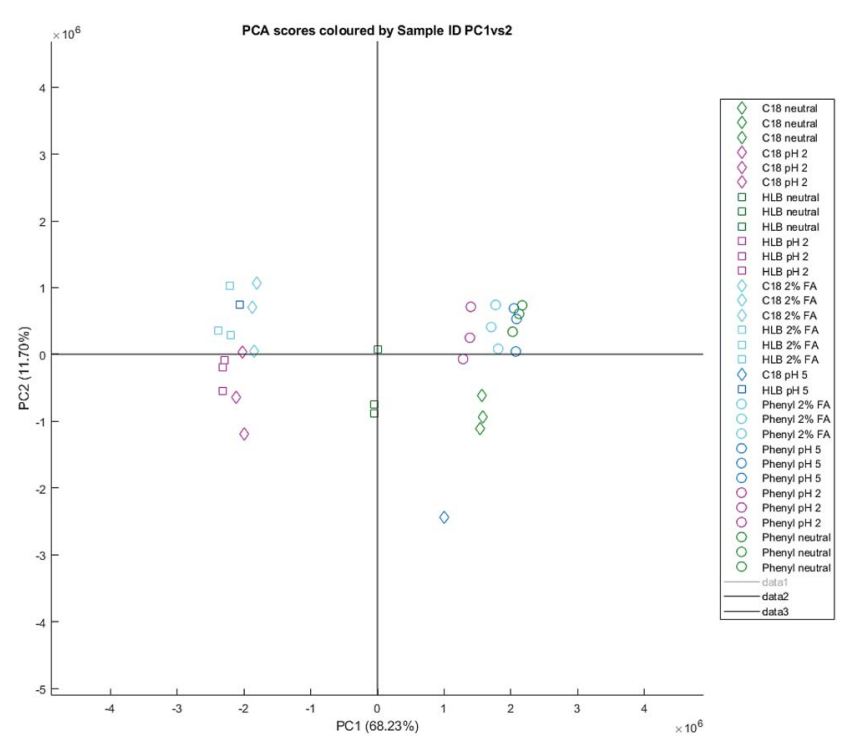

Fig. 3 A PCA scores plot based on reversed phase SPE elutions of natural urine, PC1 $(68.23 \%)$ vs. PC2 (16.76\%). 
Table 5 Natural urine reversed phase elutions, PC1 assignments

\begin{tabular}{|c|c|c|c|}
\hline Peak (ppm) & Assignment & $\begin{array}{l}\text { Pearson's } \\
\text { correlation }\end{array}$ & Phenyl correlation \\
\hline $9.13(\mathrm{~s})$ & Trigonelline & 0.78 & Positive \\
\hline $8.84(\mathrm{t})$ & Trigonelline & 0.77 & Positive \\
\hline$[8.45(\mathrm{~d})]$ & Quinolinate & $*$ & Negative \\
\hline$[8.34(\mathrm{~d})]$ & $N$-Methyl-2-pyridone-5-carboxamide & $*$ & Negative \\
\hline $8.09(\mathrm{t})$ & Trigonelline & 0.76 & Positive \\
\hline $8.03(\mathrm{~s})$ & 3-Methylxanthine & 0.84 & Negative \\
\hline $8.00(\mathrm{~s})$ & Phenylacetyl-glutamine $^{17}$ & 0.91 & Negative \\
\hline 7.97 (dd) & $N$-Methyl-2-pyridone-5-carboxamide & 0.98 & Negative \\
\hline $7.94(s)$ & Unknown I & 0.77 & Negative \\
\hline 7.91 (d?) & Unknown J & 0.93 & Negative \\
\hline 7.87 (d) & Unknown K & 0.90 & Negative \\
\hline $7.84(\mathrm{dd})$ & Hippurate & 0.98 & Negative \\
\hline 7.77 (d?) & 4-Hydroxyhippurate? & 0.92 & Negative \\
\hline 7.70 (dd) & 2-Furoylglycine & 0.92 & Negative \\
\hline $7.64(\mathrm{tt})$ & Hippurate & 0.98 & Negative \\
\hline $7.56(t)$ & Hippurate & 0.99 & Negative \\
\hline 7.46 (dd) & Quinolate & 0.93 & Negative \\
\hline $7.43(\mathrm{~m})$ & Phenylacetyl-glutamine & 0.97 & Negative \\
\hline 7.37 (m) & Phenylacetyl-glutamine & 0.97 & Negative \\
\hline $7.30(t ?)$ & Unknown L & 0.97 & Negative \\
\hline $7.24(\mathrm{~d})$ & Unknown M & 0.96 & \\
\hline 7.19 (d) & 2-Furoylglycine & 0.92 & Negative \\
\hline 7.17 (d) & 4-Hydroxyphenyl-acetate & 0.94 & Negative \\
\hline $7.12(\mathrm{ddd})$ & Unknown $\mathbf{N}$ & 0.96 & Negative \\
\hline $7.07(\mathrm{~m})$ & Unknown $\mathrm{O}$ & 0.98 & Negative \\
\hline 6.98 (d) & 4-Hydroxyhippurate? & 0.94 & Negative \\
\hline $6.93(\mathrm{t})$ & Unknown $P$ & 0.89 & Negative \\
\hline $6.87(d)$ & 4-Hydroxyphenyl-acetate & 0.95 & Negative \\
\hline 6.68 (d) & $N$-Methyl-2-pyridone-5-carboxamide & 0.97 & Negative \\
\hline $6.64(\mathrm{dd})$ & 2-Furoylglycine & 0.86 & Negative \\
\hline $4.19(\mathrm{td})$ & Phenylacetyl-glutamine & 0.98 & Negative \\
\hline $4.06(\mathrm{~s})$ & Creatinine & 0.55 & Positive \\
\hline $4.01(\mathrm{dd})$ & Phenylalanine & 0.86 & Negative \\
\hline 3.97 (d) & Hippurate & 0.98 & Negative \\
\hline $3.96(\mathrm{~s})$ & 4-Hydroxyhippurate? & 0.97 & Negative \\
\hline $3.94(\mathrm{~s} ?)$ & Unknown Q & 0.99 & Negative \\
\hline $3.93(\mathrm{~s})$ & (2-Furoylglycine) & 0.93 & Negative \\
\hline $3.92(\mathrm{~s} ?)$ & Unknown R & 0.96 & Negative \\
\hline 3.87 (s/t?) & Unknown S & 0.96 & Negative \\
\hline $3.70(\mathrm{~s})$ & Unknown T & 0.96 & Negative \\
\hline 3.67 (d) & Unknown U & 0.97 & Negative \\
\hline $3.65(\mathrm{~s})$ & $N$-Methyl-2-pyridone-5-carboxamide & 0.98 & Negative \\
\hline $3.63(\mathrm{~s})$ & Unknown $\mathbf{T}$ & 0.98 & Negative \\
\hline $3.54(\mathrm{~s})$ & Unknown V & 0.92 & Negative \\
\hline $3.53(\mathrm{~s})$ & Unknown W & 0.99 & Negative \\
\hline $3.49(\mathrm{~s})$ & Unknown X & 0.88 & Negative \\
\hline $3.48(\mathrm{~s})$ & Unknown Y & 0.96 & Negative \\
\hline $3.45(\mathrm{~s})$ & Unknown Z & 0.98 & Negative \\
\hline $3.34(\mathrm{~s})$ & Unknown AA & 0.96 & Negative \\
\hline $3.32(\mathrm{~s})$ & Unknown AB & 0.96 & Negative \\
\hline $3.30(\mathrm{~s})$ & Unknown AC & 0.97 & Negative \\
\hline $3.27(\mathrm{~s})$ & Unknown AD & 0.67 & Positive \\
\hline
\end{tabular}


Table 5 (Contd.)

\begin{tabular}{llll}
\hline & & $\begin{array}{l}\text { Pearson's } \\
\text { correlation }\end{array}$ & Phenyl correlation \\
\hline Peak (ppm) & Assignment & 0.98 & Negative \\
$3.17(\mathrm{~s})$ & Unknown AE & 0.79 & Positive \\
$3.11(\mathrm{~s})$ & Unknown AF & 0.55 & Positive \\
$3.05(\mathrm{~s})$ & Creatinine & 0.91 & Negative \\
$3.00(\mathrm{~s})$ & Unknown AG & 0.93 & Negative \\
$2.71(\mathrm{~s} ?)$ & Unknown AH & 0.87 & Negative \\
$2.69(\mathrm{~s} ?)$ & Unknown AI & 0.89 & Negative \\
$2.68(\mathrm{~s} ?)$ & Unknown AJ & 0.88 & Negative \\
$2.67(\mathrm{~s} ?)$ & Unknown AK & 0.92 & Negative \\
$2.63(\mathrm{~d})$ & Unknown T & 0.78 & Negative \\
$2.35(\mathrm{~s})$ & Unknown AL & 0.98 & Negative \\
$2.27(\mathrm{t} ?)$ & Phenylacetyl-glutamine & 0.98 & Negative \\
$2.12(\mathrm{~m} ?)$ & Phenylacetyl-glutamine & 0.98 & Negative \\
$1.93(\mathrm{~m} ?)$ & Phenylacetyl-glutamine & 0.82 & Negative \\
$1.31(\mathrm{~m})$ & Valerate & 0.92 & Negative \\
$0.94(\mathrm{~m} ?)$ & Unknown AM & 0.92 & Negative \\
$0.89(\mathrm{t})$ & Valerate & & \\
& & &
\end{tabular}

elutions than with acidified $\mathrm{C}_{18}$ or even HLB neutral elutions. The NMR signals responsible for the separation between reversed phase elutions are tabulated in Table 5 - it also notes whether the annotated metabolites or unknowns are positively correlated with phenyl cartridges (and hence retained by phenyl), or are negatively correlated (and hence retained by $\mathrm{C}_{18} / \mathrm{HLB}$ ). Signals marked with an asterisk * are not visible in the loadings plot, but can be observed with manual inspection of the spectra.

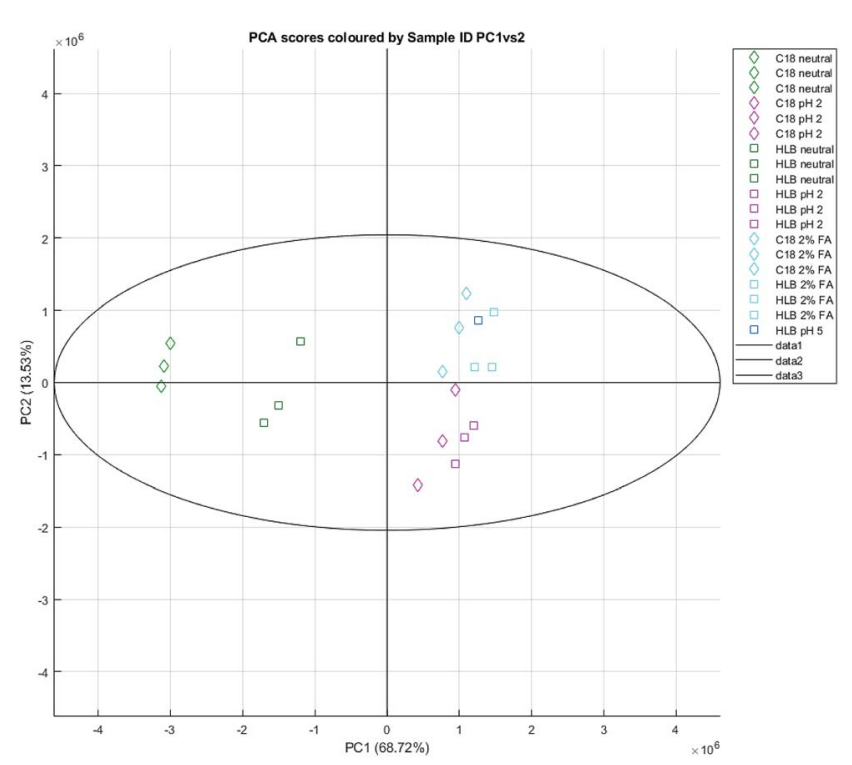

Fig. 4 A PCA scores plot based on $\mathrm{C}_{18}$ and HLB SPE elutions of natural urine, PC1 (68.72\%) vs. PC2 (13.53\%). 
Table 6 Natural urine $\mathrm{C}_{18}$ and HLB elutions, PC1 assignments

\begin{tabular}{|c|c|c|c|}
\hline Peak (ppm) & Assignment & $\begin{array}{l}\text { Pearson's } \\
\text { correlation }\end{array}$ & HLB correlation \\
\hline $8.05(\mathrm{~m} ?)$ & Unknown AN & 0.87 & Positive \\
\hline 7.97 (br s?) & Unknown AO & 0.91 & Positive \\
\hline 7.97 (dd) & $N$-Methyl-2-pyridone-5-carboxamide & 0.91 & Negative \\
\hline 7.96 (dd) & $N$-Methyl-2-pyridone-5-carboxamide & 0.96 & Positive \\
\hline $7.94(s)$ & Unknown I & 0.70 & Positive \\
\hline $7.93(\mathrm{~s})$ & Unknown AP & 0.83 & Negative \\
\hline $7.92(\mathrm{~s})$ & Unknown AQ & 0.82 & Negative \\
\hline $7.91(\mathrm{~s})$ & Unknown AR & 0.91 & Positive \\
\hline $7.91(\mathrm{~s})$ & Unknown AS & 0.61 & Negative \\
\hline $7.90(\mathrm{~s})$ & Unknown AT & 0.84 & Positive \\
\hline 7.87 (d) & Unknown K & 0.83 & Positive \\
\hline $7.84(\mathrm{dd})$ & Hippurate & 0.91 & Positive \\
\hline 7.76 (d?) & 4-Hydroxyhippurate? & 0.88 & Positive \\
\hline 7.70 (dd) & 2-Furoylglycine & 0.81 & Positive \\
\hline $7.64(\mathrm{tt})$ & Hippurate & 0.99 & Positive \\
\hline $7.56(\mathrm{t})$ & Hippurate & 0.99 & Positive \\
\hline $7.43(\mathrm{~m})$ & Phenylalanine & 0.95 & Positive \\
\hline 7.37 (m) & Phenylalanine & 0.95 & Positive \\
\hline $7.31(\mathrm{~s})$ & Unknown AU & 0.83 & Positive \\
\hline $7.30(\mathrm{t})$ & Unknown L & 0.92 & Positive \\
\hline 7.19 (d) & 2-Furoylglycine & 0.80 & Positive \\
\hline 7.17 (d) & 4-Hydroxyphenyl-acetate & 0.85 & Positive \\
\hline 7.12 (ddd) & Unknown $\mathbf{N}$ & 0.96 & Positive \\
\hline $6.98(d)$ & 4-Hydroxyhippurate? & 0.85 & Positive \\
\hline $6.87(\mathrm{~d})$ & 4-Hydroxyphenyl-acetate & 0.90 & Positive \\
\hline $6.81(\mathrm{~s} / \mathrm{t} ?)$ & Unknown AV & 0.92 & Positive \\
\hline $6.68(\mathrm{~d})$ & $N$-Methyl-2-pyridone-5-carboxamide & 0.92 & Positive \\
\hline $6.67(d)$ & $N$-Methyl-2-pyridone-5-carboxamide & 0.91 & Negative \\
\hline $4.19(\mathrm{td})$ & Phenylacetylglutamine & 0.96 & Positive \\
\hline $4.06(\mathrm{~s})$ & Creatinine & 0.80 & Negative \\
\hline 3.97 (d) & Hippurate & 0.99 & Positive \\
\hline $3.96(\mathrm{~s})$ & Unknown AW & 0.94 & Positive \\
\hline 3.94 (d?) & Unknown AX & 0.92 & Negative \\
\hline 3.93 (d?) & Unknown AY & 0.95 & Positive \\
\hline $3.93(\mathrm{~s})$ & Unknown AZ & 0.88 & Positive \\
\hline 3.87 (s/t?) & Unknown $\mathrm{T}$ & 0.98 & Positive \\
\hline $3.70(\mathrm{~s})$ & Unknown BA & 0.98 & Positive \\
\hline 3.67 (d?) & Unknown BB & 0.95 & Positive \\
\hline $3.65(\mathrm{~s})$ & $N$-Methyl-2-pyridone-5-carboxamide & 0.92 & Negative \\
\hline $3.65(\mathrm{~s})$ & $N$-Methyl-2-pyridone-5-carboxamide & 0.97 & Positive \\
\hline $3.63(\mathrm{~s})$ & Unknown $T$ & 0.97 & Positive \\
\hline $3.53(\mathrm{~s})$ & Unknown BC & 0.79 & Negative \\
\hline $3.53(\mathrm{~s})$ & 3-Methylxanthine? & 0.95 & Positive \\
\hline $3.49(\mathrm{~s})$ & Unknown BD & 0.81 & Negative \\
\hline $3.49(\mathrm{~s})$ & Unknown BE & 0.84 & Positive \\
\hline $3.48(\mathrm{~s})$ & Unknown BF & 0.90 & Positive \\
\hline $3.46(\mathrm{~s})$ & Unknown BG & 0.93 & Positive \\
\hline $3.45(\mathrm{~s})$ & Unknown BH & 0.88 & Positive \\
\hline $3.36(\mathrm{~s})$ & Unknown BI & 0.87 & Negative \\
\hline $3.35(\mathrm{~s} ?)$ & Unknown BJ & 0.86 & Negative \\
\hline 3.34 (s?) & Unknown BK & 0.95 & Positive \\
\hline 3.33 (s?) & Unknown BL & 0.83 & Negative \\
\hline
\end{tabular}


Table 6 (Contd.)

\begin{tabular}{llll}
\hline & & $\begin{array}{l}\text { Pearson's } \\
\text { correlation }\end{array}$ & HLB correlation \\
\hline $3.32(\mathrm{~s} ?)$ & Unknown BM & 0.93 & Positive \\
$3.30(\mathrm{~s} ?)$ & Unknown BN & 0.96 & Positive \\
$3.27(\mathrm{~s})$ & TMAO & 0.80 & Negative \\
$3.17(\mathrm{~s})$ & Unknown BO & 0.88 & Negative \\
$3.17(\mathrm{~s})$ & Unknown BP & 0.95 & Positive \\
$3.05(\mathrm{~s})$ & Creatinine & 0.80 & Negative \\
$2.27(\mathrm{t})$ & Phenylacetyl-glutamine & 0.97 & Positive \\
$2.12(\mathrm{~m})$ & Phenylacetyl-glutamine & 0.97 & Positive \\
$0.93(\mathrm{~m})$ & Phenylacetyl-glutamine & 0.97 & Positive
\end{tabular}

Clustering can be observed forming an almost linear scale for $\mathrm{C}_{18}$ and HLB cartridges (Fig. 4) - with $\mathrm{C}_{18}$ neutral elutions at one end, acidified elutions at the other, and HLB neutral elutions in between. Many of the assigned peaks (Table 6) in the aromatic region are caused by differences in chemical shift between identical compounds (likely due to $\mathrm{pH}$ differences) - for example, $\mathrm{N}$-methyl-2pyridone-5-carboxamide (2PY) is significantly correlated both positively and negatively with phenyl elutions (Fig. 5), as its spectral peaks undergo chemical shifting due to $\mathrm{pH}$ differences in different experiments.

The PC3 vs. PC4 plot in the all-elutions structure also demonstrated clustering (Fig. 6) of the PBA elutions, positively correlated with the PC4 dimension. The PC4 loadings hence closely resemble the averaged spectra from the PBA elutions - the metabolites (Table 7) being mostly represented by mannitol and $\mathrm{N}$ methylnicotinamide.

\section{Artificial urine}

A similar PCA can be constructed for reversed phase elutions utilising artificial urine - again, there is separation across the first component, demonstrating

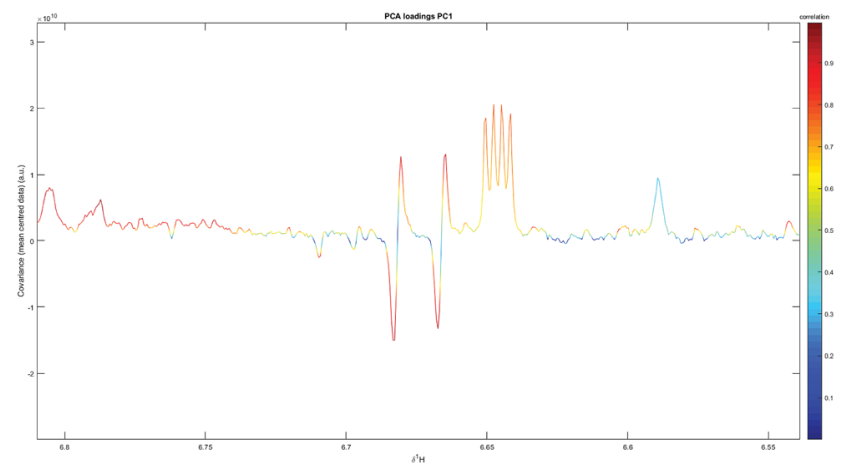

Fig. 5 The PC1 loadings plot for $\mathrm{C}_{18} / \mathrm{HLB}$ elutions, demonstrating both positive and negative correlation of $2 \mathrm{PY}$. 


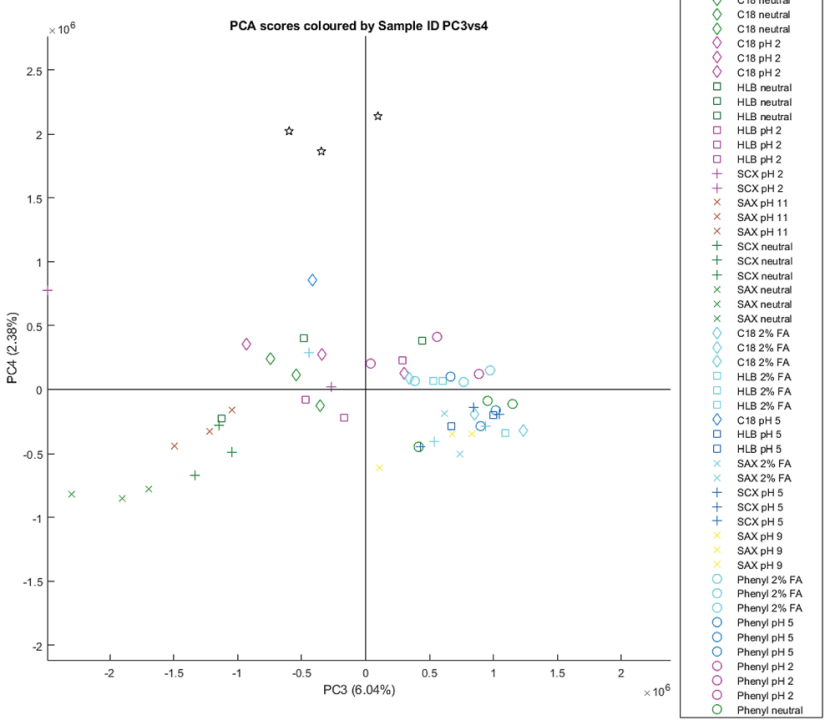

Fig. 6 A PCA scores plot based all SPE elutions of natural urine, PC3 $(6.04 \%)$ vs. PC4 (2.38\%).

a notable difference between phenyl and $\mathrm{C}_{18} / \mathrm{HLB}$ retention profiles. As with the natural urine elutions, the loadings (Table 8) can be annotated to demonstrate the most important spectral differences separating different methods.

Table 7 Natural urine all elutions, PC4 assignments

\begin{tabular}{lll}
\hline Peak (ppm) & Assignment & $\begin{array}{l}\text { Pearson's } \\
\text { correlation }\end{array}$ \\
\hline $9.29(\mathrm{~s})$ & N-Methylnicotinamide & 0.82 \\
$8.97(\mathrm{~d})$ & $N$-Methylnicotinamide & 0.72 \\
$8.90(\mathrm{~d})$ & $N$-Methylnicotinamide & 0.71 \\
$8.79(\mathrm{~d})$ & Unknown BQ & 0.69 \\
{$[8.53(\mathrm{t})]$} & Unknown BQ & $*$ \\
$8.06(\mathrm{t} ?)$ & Unknown BQ & 0.63 \\
$7.68(\mathrm{~s})$ & Unknown BR & 0.88 \\
$5.85(\mathrm{~d})$ & Unknown BS & 0.49 \\
{$[4.31(\mathrm{t})]$} & Unknown BS & $*$ \\
$4.16(\mathrm{t})$ & Unknown BS & 0.53 \\
$4.11(\mathrm{~s})$ & Unknown BT & 0.68 \\
$4.02(\mathrm{t})$ & Unknown BU & 0.73 \\
$3.89(\mathrm{~d})$ & Mannitol & 0.86 \\
$3.87(\mathrm{~d})$ & Mannitol & 0.87 \\
$3.82(\mathrm{~s})$ & Mannitol & 0.86 \\
$3.80(\mathrm{~s})$ & Mannitol & 0.84 \\
$3.77(\mathrm{~m})$ & Mannitol & 0.87 \\
$3.67(\mathrm{~d})$ & Unknown BV & 0.87 \\
$3.69(\mathrm{dd})$ & Mannitol & 0.86 \\
$3.20(\mathrm{~s})$ & Unknown BW & 0.79 \\
$2.76(\mathrm{t})$ & Ethanolamine? & 0.76 \\
$2.72(\mathrm{~s})$ & DMA & 0.85 \\
$2.01(\mathrm{~s})$ & Acetamide? & 0.82 \\
& &
\end{tabular}


Table 8 Artificial urine reversed phase elutions, PC1 assignments

\begin{tabular}{|c|c|c|c|}
\hline Peak (ppm) & Assignment & $\begin{array}{l}\text { Pearson's } \\
\text { correlation }\end{array}$ & Phenyl correlation \\
\hline $9.13(\mathrm{~s})$ & Trigonelline & 0.95 & Positive \\
\hline $8.84(\mathrm{t})$ & Trigonelline & 0.97 & Positive \\
\hline $8.09(\mathrm{t})$ & Trigonelline & 0.92 & Positive \\
\hline 7.97 (d) & Hippurate & 0.97 & Negative \\
\hline $7.88(\mathrm{~m})$ & Benzoate & 1.00 & Negative \\
\hline $7.84(\mathrm{dt})$ & Hippurate & 1.00 & Negative \\
\hline $7.78(\mathrm{t})$ & Hippurate & 0.97 & Negative \\
\hline $7.70(\mathrm{t})$ & Hippurate & 0.97 & Negative \\
\hline $7.64(\mathrm{tt})$ & Hippurate & 1.00 & Negative \\
\hline $7.56(\mathrm{~m})$ & Hippurate & 1.00 & Negative \\
\hline 7.49 (m) & Benzoate & 1.00 & Negative \\
\hline $7.44(\mathrm{tt})$ & Phenylalanine & 0.99 & Negative \\
\hline $7.38(\mathrm{tt})$ & Phenylalanine & 0.99 & Negative \\
\hline $7.34(\mathrm{~m})$ & Phenylalanine & 0.99 & Negative \\
\hline $7.17(\mathrm{dt})$ & 4-Hydroxyphenyl-acetate & 0.93 & Negative \\
\hline $6.87(\mathrm{dt})$ & 4-Hydroxyphenyl-acetate & 0.93 & Negative \\
\hline $6.59(\mathrm{~s})$ & trans-Aconitate & 0.85 & Negative \\
\hline $4.00(q)$ & Phenylalanine & 0.96 & Negative \\
\hline 3.97 (d) & Hippurate & 0.99 & Negative \\
\hline 3.30 (d) & Phenylalanine? & 0.97 & Negative \\
\hline 3.27 (s) & TMAO & 0.91 & Positive \\
\hline $3.13(\mathrm{~m})$ & Phenylalanine? & 0.99 & Negative \\
\hline $3.05(\mathrm{~s})$ & Creatine? & 0.93 & Positive \\
\hline $2.66(d)$ & Citrate & 0.91 & Negative \\
\hline $2.55(\mathrm{~d})$ & Citrate & 0.94 & Negative \\
\hline $2.41(\mathrm{~s})$ & Succinate & 0.84 & Negative \\
\hline
\end{tabular}

Here, artificial urine was used to demonstrate that a mixture of representative compounds can be used to estimate the retention capacity of cartridges without using natural urine - as with the natural urine reversed phase elutions, metabolites such as hippurate can be shown to be retained on $\mathrm{C}_{18} / \mathrm{HLB}$, but not on phenyl cartridges; similarly, trigonelline can be shown to be retained on phenyl, but not on $\mathrm{C}_{18} / \mathrm{HLB}$. This allows for greater control over future SPE experiments aimed at characterising retention profiles of SPE cartridges.

\section{Discussion}

The guiding philosophy behind this use of SPE-NMR suggests that not only each cartridge, but each $\mathrm{pH}$ and solvent system utilised in a given experiment, would result in different retention profiles. These retention profiles can be classified either through annotation of a selection of common metabolites from different compound classes, or using a more holistic approach, determining the compounds more likely to be retained under different conditions through data treatment. Selective use of methods can then be utilised to reduce peak overlap and aid metabolite identification. One example of this is displayed in Fig. 7; interferences in the 'raw' pooled urine sample are removed by the use of a PBAbased method to clearly reveal mannitol. 

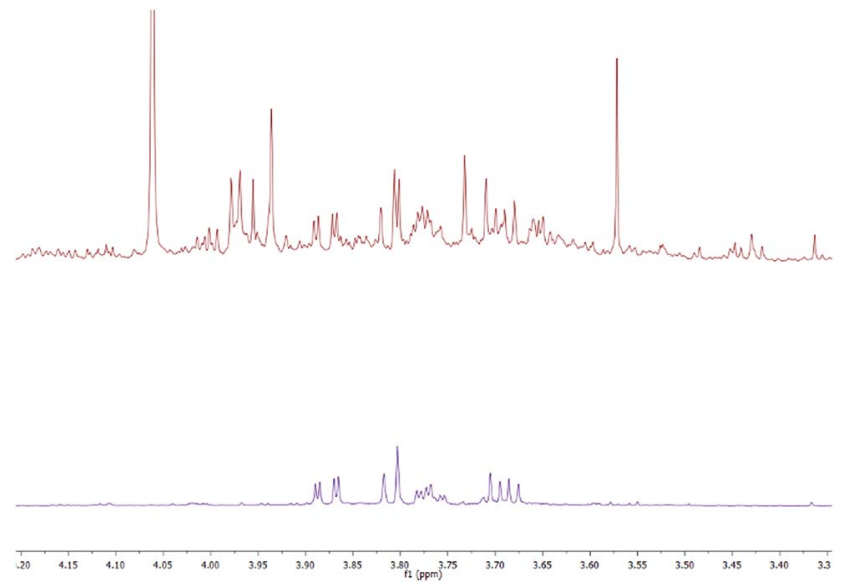

Fig. 7 Comparison of the 3.30-4.15 ppm region of the $600 \mathrm{MHz}$ NMR spectra of the pooled urine sample (top) and PBA SPE-treated urine (bottom), the latter revealing only mannitol peaks.

Annotation of a selection of common metabolites is facile and provides immediate and useful information of individual compounds. Ideally, this could be done using a list of metabolites representative of the compound classes generally found in human urine; unfortunately, the partial identification of the human urinary metabolome hinders the creation of a fully representative sample. Additionally, the natural rate of occurrence of metabolites may make a representation of an 'average' sample difficult or even impossible. Peak intensities can also be impacted by NMR shimming, peak overlap, and pH changes - all of which can affect the intensity recorded. Despite these shortcomings, general trends can be established by considering functional groups and structural commonalities between compound classes.

Clustering in PCA plots can be used to demonstrate substantial differences between datasets. The Strong Cation Exchange (SCX) cartridge - utilising the pseudo-permanently charged phenylsulfonic derivative $\left(\mathrm{p} K_{\mathrm{a}} \approx 2.1\right)$ - provides the greatest separation between clusters when included in PCA structures, due to the ion exchange mechanisms not present in reversed phase chromatography. Ion exchange retention profiles rely heavily on $\mathrm{pH}$ control, since all compounds must have at least one positively charged atom in order to have sufficient attraction to the sorbent to be retained; hence, compounds that do not have a positive charge at physiological $\mathrm{pH}$ must be in acidic solution for retention to occur. This intrusive sample adjustment will naturally affect the chemistry of the biofluid; using acidified (or basified) conditions is, then, necessarily a trade-off between greater insight into the metabolome through retention, and authenticity of the sample itself. It is additionally feasible that compounds not normally present in the sample may be formed and retained due to the change of conditions, although this was not noted during the course of the experiments.

The importance of $\mathrm{pH}$ control is reflected in the retention capacity of SCX cartridges; the neutral $\mathrm{pH}$ retention profile is one of the least retaining methods with an estimated retention capacity of $0-$ at $\mathrm{pH} 2$, its retention capacity (14.67) is 
comparable to a more widely recognised reversed phase method, such as HLB with $2 \%$ formic acid in all steps (17.40). The compounds best retained on SCX under acidic conditions were predominantly histidine-based - with histidine, 3methylhistidine, and 1-methylhistidine being well retained at $\mathrm{pH}$ 2. Creatinine and TMAO were also present in the elutions. A cationic nitrogen atom, possibly stabilised by electron-donating groups through hyperconjugation, may serve as the most important characteristic uniting the compounds. Compounds without nitrogen-containing functional groups (such as amino acids) were generally not present in the acidified SCX elutions, although the presence of a nitrogencontaining functional group did not necessarily result in retention - for example, of the proteinogenic amino acids that were retained, only histidine was retained in any significant quantity. It may be notable that histidine has a $\mathrm{p} K_{\mathrm{a}}$ of 6.04 (pyrrolic nitrogen), far lower than the other positively charged amino acids, arginine $\left(\mathrm{p} K_{\mathrm{a}} 12.10\right)$ and lysine $\left(\mathrm{p} K_{\mathrm{a}} 10.67\right)$.

Removing ion exchange elutions from the dataset and reconstructing a PCA plot demonstrates additional separation between $\mathrm{C}_{18} / \mathrm{HLB}$ and phenyl elutions and allows for further probing into the differences between the reversed phase methods. Previous uses of phenyl cartridges in the literature have remarked on their similar retention capabilities to $\mathrm{C}_{18}$, with slightly better retention for polycyclic aromatic compounds, ${ }^{18}$ but slightly worse retention for other hydrocarbons. ${ }^{19}$ Phenyl cartridges utilise $\pi$-stacking on top of hydrophobic forces in order to provide additional retention for aromatic compounds - however, the strength of $\pi-\pi$ interactions tends to be bound between around $8-12 \mathrm{~kJ} \mathrm{~mol}^{-1}$ for benzene dimers. ${ }^{20}$ For comparison, hydrophobic forces may be up to 4 times stronger; ${ }^{21}$ hence, despite having an additional mechanism of action, the actual retention capacity for phenyl cartridges is significantly lower than that of $\mathrm{C}_{18}$ or HLB cartridges across all methods due to a weaker hydrophobic retention mechanism.

The two major compounds that were retained selectively by phenyl (but not by $\mathrm{C}_{18}$ or HLB) were trigonelline and creatinine, both nitrogen-containing heterocycles. Other compounds with phenyl functional groups - such as phenylalanine or hippurate - did not experience greater retention using phenyl cartridges, and in fact retained much less, if at all. Conversely, the cyclic metabolites retained under acidic conditions by $\mathrm{C}_{18} / \mathrm{HLB}$ - but not by phenyl - are predominantly aromatic, with both heterocycles (2-furoylglycine, quinolinate, and $\mathrm{N}$-methyl-2pyridone-5-carboxamine), and hydrocarbon rings (hippurate, phenylacetylglutamine, and $p$-hydroxyphenylacetate) present. The aromatic heterocycles here do not have the ability to form cationic nitrogen in the rings themselves, unlike the aromatic compounds retained in phenyl elutions, such as trigonelline. Short chain fatty acids such as valeric acid were also retained under non-neutral conditions. It is unclear why charged metabolites like trigonelline would be better retained on phenyl cartridges - although as the charge is positive, it is hypothetically possible that the $\pi$-electron clouds located above and below the benzene rings are able to electrostatically attract these metabolites with enough strength that they can be retained.

Out of the remaining reversed phase sorbents, the $\mathrm{C}_{18}$ and HLB cartridges are known to have similar retention profiles to each other, ${ }^{10}$ with HLB cartridges often being preferred for their tolerance to drying and the possibility for elimination of conditioning and equilibration steps. The differences between the two can be demonstrated through comparison of elutions - while the two have comparable 
retention, HLB cartridges tend to retain a slightly larger range of compounds in greater quantities. This is especially true under neutral conditions, where $\mathrm{C}_{18}$ cartridges retain relatively little. Generally speaking, as with the SCX cartridges, more acidic conditions result in greater retention - possibly due to the deionisation of silanol groups on the surface of the sorbent. However, this again comes with the trade-off of authenticity, as the acidic conditions may cause signal suppression, unwanted reactions between metabolites, or general degradation of the sample itself. Use of $2 \%$ formic acid in all steps allows for a balance between the two - while the retention does not extend as deeply as that under $\mathrm{pH} 2$ conditions, the higher $\mathrm{pH}$ environment should not be as destructive to sample authenticity, and chemical shifts caused by drastic $\mathrm{pH}$ changes should be absent. HLB and $\mathrm{C}_{18}$ under acidic conditions are powerful analytical methods that can reveal signals that are otherwise not visible - for example, unknown $\mathrm{N}$, which displays a doublet of doublet of doublets (ddd) signal at $7.12 \mathrm{ppm}$, is normally obscured by a 3-methylhistidine peak (Fig. 8). The use of reversed phase methods can hence provide additional information about the human urinary metabolome.

$\mathrm{C}_{18}$ and HLB cartridges themselves can also be differentiated from each other. On top of the obvious differences in sorbent structure (a hydrocarbon chain, compared to a polymer containing divinylbenzene), HLB cartridges are not silicabased - hence, silanol groups present in $\mathrm{C}_{18}$ cartridges are not present in HLB. These silanol groups produce secondary interactions with metabolites, commonly expressed as a weak cation exchanger, which can influence retention. This is reflected in the differences between $\mathrm{C}_{18}$ elutions under neutral and acidified conditions - at lower $\mathrm{pH}$, the silanols are generally protonated; at neutral $\mathrm{pH}$, at least some silanols are deprotonated, giving the cartridge the ability to selectively retain some cations. Indeed, observing the PCA results shows that compounds such as creatinine and TMAO are retained under neutral conditions - these compounds also being retained by the SCX cartridge under the appropriate conditions.

The final elutions to consider were those afforded from methods utilising phenylboronic acid (PBA) cartridges. PBA cartridges utilise a unique covalent

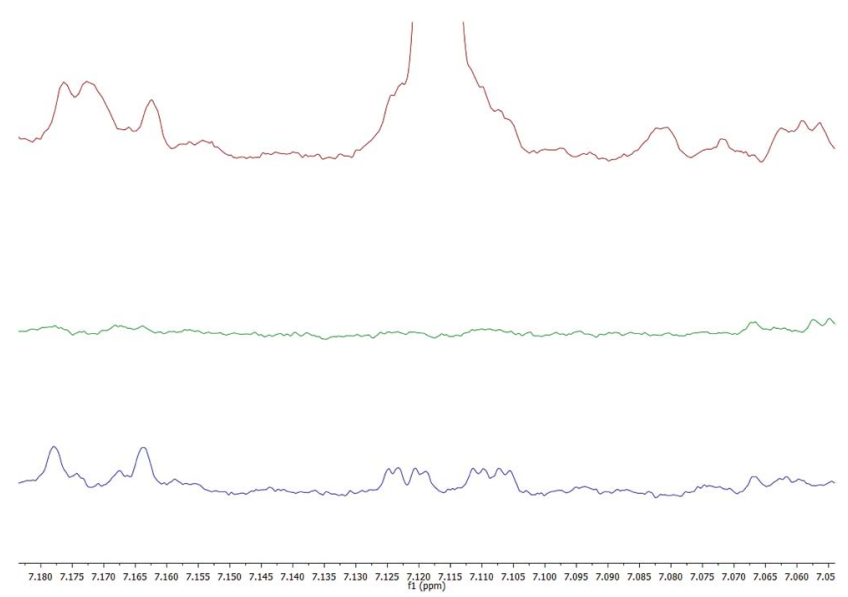

Fig. 8 Unknown N (ddd), normally obscured by 3-methylhistidine (top), is revealed after HLB SPE treatment under acidic conditions (bottom), but not under neutral conditions (middle). 
bonding mechanism in order to selectively retain diols, $\alpha$-hydroxy ketones, or any other functional groups where two unsubstituted heteroatoms are separated by at least one carbon. ${ }^{22,23}$ It is not clear whether the diols must adopt a specific isomerism for retention to occur: mannitol is heavily retained in the elutions, but contains both $\mathrm{R}$ and $\mathrm{S}$ carbon centres, as well as terminal hydroxyls which can rotate to become a given conformer - its retention hence does not give additional insight. Other compounds retained include acetate, acetamide, and $\mathrm{N}$-methylnicotinamide, a metabolite of niacin. The presence of adjacent heteroatoms does not guarantee good retention: for example, citric acid is poorly retained, despite having three carboxylate groups. There is also some retention of dimethylamine in both artificial and natural urine samples, despite it not being a diol - however, it could hypothetically be retained through a single substitution of water at the boronate, rather than through a double-substitution, as is normally the case.

\section{Conclusions}

We have demonstrated and compared the use of different SPE methods for the retention of different compound classes within the human urinary metabolome. Different retention profiles can give unique insight into the metabolome by revealing metabolite peaks in NMR spectroscopy that had previously been obscured by peak overlap - SPE can hence be used to either remove the suppressing metabolite(s) or to isolate the unknown metabolite itself. These retention profiles can be differentiated based on their retention of not only individual metabolites, but of broader subclasses of compounds united by their structure commonalities, including shared functional groups. Hence, different methods can be utilised in order to give greater control over the annotation process. On top of the metabolites identified by comparison to metabolite databases and available literature, several unknowns were annotated in the elutions - further experiments and comparisons to an authentic chemical reference will be required to identify them to a level 1 standard. ${ }^{16}$ There also exists the possibility to study washes from the SPE experiments further, as well as to use multiple SPE methods in series, in order to narrow down a set of retained compounds even further. Finally, it may be possible to transfer these methods to an automated SPE system for more highthroughput analysis. All of these will hopefully broaden our understanding of the human urinary metabolome, and hence our understanding of biomarkers and the disease states and phenotypes that they represent.

\section{Conflicts of interest}

The authors register no conflicts of interest.

\section{Acknowledgements}

Author Dan McGill is supported by the Bruker Corporation and the Stratified Medicine Graduate Training Programme in Systems Medicine and Spectroscopic Profiling (STRATiGRAD). The authors would like to thank Rose Tolson (Imperial College London) for running the polyethylene glycol screening and Gordon Haggart (Imperial College London) for his assistance with troubleshooting the 
IMPaCTS toolbox. Elena Chekmeneva was supported by the NIHR Imperial Biomedical Research Centre.

\section{Notes and references}

1 P. Elliott, et al., Urinary metabolic signatures of human adiposity, Sci. Transl. Med., 2015, 7, 285ra62.

2 J. K. Nicholson and I. D. Wilson, High resolution proton magnetic resonance spectroscopy of biological fluids, Prog. Nucl. Magn. Reson. Spectrosc., 1989, 21, 449-501.

3 S. L. Robinette, J. C. Lindon and J. K. Nicholson, Statistical Spectroscopic Tools for Biomarker Discovery and Systems Medicine, Anal. Chem., 2013, 85, 52975303.

4 J. M. Posma, et al., Integrated Analytical and Statistical Two-Dimensional Spectroscopy Strategy for Metabolite Identification: Application to Dietary Biomarkers, Anal. Chem., 2017, 89, 3300-3309.

5 K. Bingol and R. Brüschweiler, NMR/MS Translator for the Enhanced Simultaneous Analysis of Metabolomics Mixtures by NMR Spectroscopy and Mass Spectrometry: Application to Human Urine, J. Proteome Res., 2015, 14, 2642-2648.

6 S. Bouatra, et al., The Human Urine Metabolome, PLoS One, 2013, 8, e73076.

7 L. W. Sumner, et al., Proposed minimum reporting standards for chemical analysis Chemical Analysis Working Group (CAWG) Metabolomics Standards Initiative (MSI), Metabolomics, 2007, 3, 211-221.

8 I. D. Wilson and J. K. Nicholson, Solid phase extraction chromatography and NMR spectroscopy (SPEC-NMR) for the rapid identification of drug metabolites in urine, J. Pharm. Biomed. Anal., 1988, 6, 151-165.

9 W. Yang, Y. Wang, Q. Zhou and H. Tang, Analysis of human urine metabolites using SPE and NMR spectroscopy, Sci. China, Ser. B: Chem., 2008, 51, 218-225.

10 D. M. Jacobs, L. Spiesser, M. Garnier, N. De Roo, F. Van Dorsten, B. Hollebrands, E. Van Velzen, R. Draijer and J. Van Duynhoven, SPE-NMR metabolite sub-profiling of urine, Anal. Bioanal. Chem., 2012, 404, 2349-2361.

11 A. C. Dona, et al., Precision High-Throughput Proton NMR Spectroscopy of Human Urine, Serum, and Plasma for Large-Scale Metabolic Phenotyping, Anal. Chem., 2014, 86, 9887-9894.

12 Imperial Metabolic Profiling and Chemometrics Toolbox (IMPaCTS), available at: 10.5281/zenodo.803330.

13 F. Dieterle, A. Ross, G. Schlotterbeck and H. Senn, Probabilistic Quotient Normalization as Robust Method to Account for Dilution of Complex Biological Mixtures. Application in ${ }^{1} \mathrm{H}$ NMR Metabonomics, Anal. Chem., 2016, 78, 4281-4290.

14 The Human Urine Metabolome Database, available at: http:// www.urinemetabolome.ca/.

15 P. G. Takis, H. Schäfer, M. Spraul and C. Luchinat, Deconvoluting interrelationships between concentrations and chemical shifts in urine provides a powerful analysis tool, Nat. Commun., 2017, 8, 1662.

16 L. W. Sumner, et al., Proposed quantitative and alphanumeric metabolite identification metrics, Metabolomics, 2014, 10, 1047-1049. 
$17 \mathrm{~W}$. Barton, et al., The microbiome of professional athletes differs from that of more sedentary subjects in composition and particularly at the functional metabolic level, Gut, 2017, 67, gutjnl-2016-313627.

18 R. Marcé and F. Borrull, Solid-phase extraction of polycyclic aromatic compounds, J. Chromatogr. A, 2000, 885, 273-290.

19 M.-C. Hennio, Solid-phase extraction: method development, sorbents, and coupling with liquid chromatography, J. Chromatogr. A, 1999, 856, 3-54.

20 M. O. Sinnokrot, E. F. Valeev and C. D. Sherrill, Estimates of the Ab Initio Limit for $\pi-\pi$ Interactions: The Benzene Dimer, J. Am. Chem. Soc., 2002, 124, 1088710893.

21 R. H. Petrucci, F. G. Herring, J. D. Madura and C. Bissonnette, General Chemistry: Principles and Modern Applications, Prentice Hall, 2016.

22 M. Tugnait, F. Y. K. Ghauri, I. D. Wilson and J. K. Nicholson, NMR-monitored solid-phase extraction of phenolphthalein glucuronide on phenylboronic acid and C18 bonded phases, J. Pharm. Biomed. Anal., 1991, 9, 895-899.

23 P. Martin, B. Leadbetter and I. D. Wilson, Immobilized phenylboronic acids for the selective extraction of $\beta$-blocking drugs from aqueous solution and plasma, J. Pharm. Biomed. Anal., 1993, 11, 307-312. 